\title{
The Studies on the Kidney Homotransplantation
}

I. The Elucidation of the Systemic Toxic Syndrome Developed in the Recipient

By

\author{
Yoshinobu Ishikawa, Keizo Miura, Hozue Kimura and \\ (石川義信) \\ （三浦慶造）（木村秀枝） \\ Koichi Mizutani \\ (水谷 恒 $一$ ) \\ From the Katsura Surgical Service, Tohoku University, Faculty of \\ Medicine, Sendai; Director: Prof. S. T. Katsura
}

(Received for publication, May 10, 1957)

In spite of few exceptional clinical successful reports on homotransplantation of the kidney ${ }^{11-4)}$, everybody who is engaged in this work agrees that the homotransplanted kidney would stop its function sooner or later and become necrotic ${ }^{1,2,2,5)-77}$. The most prevailing theory on its etiology is the aquired immunity theory ${ }^{51,71,8)}$ which has a fatal defect which cannot demonstrate cyto-toxic homologous antibody in vitro. On the other hand, systemic toxic syndrome ${ }^{5,9), 10)}$, which appears in the recipient homotransplanted kidney is an important problem which cannot be overlooked. However, no extensive study could be found as far as we could determine, probably owing to its mysterious nature. While the authors performed homotransplantation of the kidney to study the necrotizing process of the transplanted kidney, and organic pathology was found in the recipient kidney and liver as similar as that in the transplanted kidney. This new observation is reported.

\section{EXPERIMENTAL}

\section{Method}

Healthy mongrol dogs were selected as a pair of dog which showed negative agglutination test by cross matching test of blood at room temperature for 30-60 minutes. Both donor and recipient were injected with $2 \%$ morphine for premedication. 20-60 minutes later, they were anesthetized with intravenous injection of $0.3-0.5 \mathrm{gm}$. pentothal sodium. Following removal of the donnor kidney, it was irrigated with 200-300 cc. physiologic saline solition under a falling pressure of $110-120 \mathrm{~mm} . \mathrm{H}_{2} \mathrm{O}$ and then transplanted in the cervical region of the donor. The experiment was divided into two groups; Group 1; Removal of the recipient kidneys 
and Group 2 ; Nonremoval of the recipient kidneys. The recipient ureter was anastomosed to the recipient skin at the neck. Transplanted kidney without function, recipient organs from a dead dog, transplanted kidney and recipient organs which were autopsied at necessary time were all fixed in $10 \%$ formalin. Histological study was made on them after being stained with haematoxylin eosin (abbr. H-E staining), van Gieson's method (abbr. V staining) and methylene-green pyronin (abbr. M-P staining).

\section{Results}

I. Group 1: Bilateral nephrectomy of the recipient after homologous transplantation of a kidney

Experiment: Following homologous transplantation of the kidney, dogs became apathic, anorexic, and weak. Some just lied on the floor. Blood was taken for test everyday. 5 days after operation, serum showed yellow appearance suggesting latent jaundice. No haemolysis was, however, noticed. This symptom was considered to be due to uremia, but no special test was carried out. Urination from the transplanted kidney continued from 1-8 days. The kidney became hyperaemic and swollen. Its histology by M-P staining showed interstitial nephritis accompanied with pyroninophilic cells, which resembled plasma cells in which cytoplasma was red stained (Fig. 1). Such pathological findings did not only

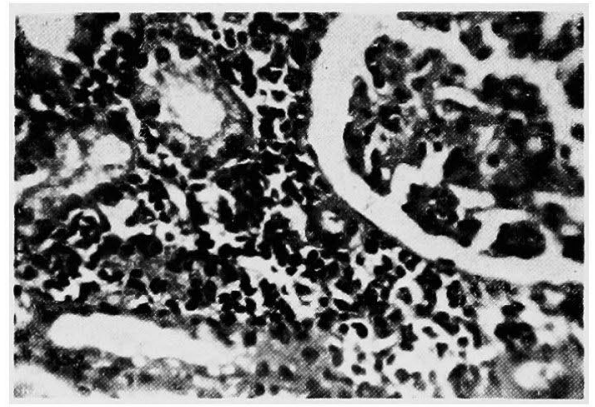

Fig. 1. Exp. No. DH 35. Homotransplanted kidney after 8 days. M-P staining. $\times 300$. Periglomerular accumulation of round cells including many pyroninophilic cells. Glomerulus is normal.

occur in the transplanted kidney, but was also seen in the recipient liver; stagnation of blood, central necrosis, infiltration of pyroninophilic cells around the blood vessels in the Portal space were observed (Fig. 2, 3).

II. Group: No Nephrectomy after homotransplantation of a kidney

Experiment: As the recipient own kidney was left in the body, the dog did not die. The transplanted kidney was not touched till it became necrotic and absorbed. The dog was not vigorous till the urination from 


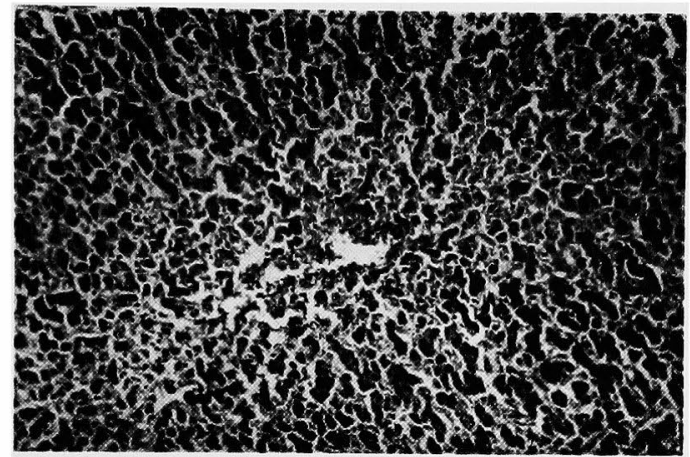

Fig. 2. Exp. No. DH 8. Recipient liver after 8 days. H-E staining. $\times 60$. Stasis, hyperaemia, central degeneration and necrosis. Liver cells are in degeneration and necrosis. Liver cells are in dissociation.

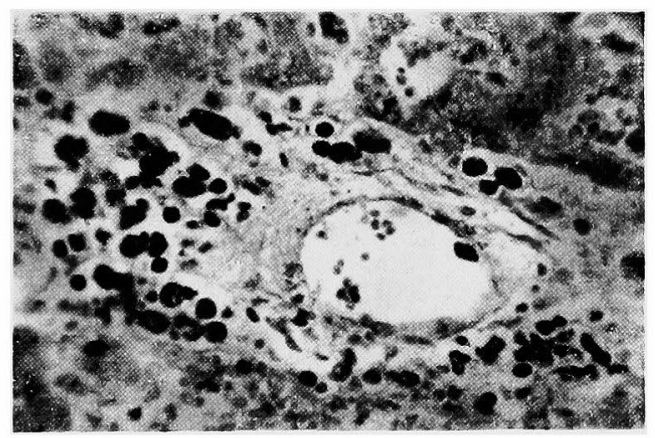

Fig. 3. Exp. No. DH 8. Recipient liver after 8 days. M-P staining. $\times 300$. Perivascular accumulation of pyroninophilic cells in the portal space.

the transplanted kidney stopped. Soon after the renal function of the transplanted kidney ceased, the dog became vigorous and walked around which seemed to be a interesting observation. The collected serum started to show yellow appearance after 5 days. When the absorbed transplanted kidney was carefully studied (Fig. 4), the urinary tubules in the glomeruli was found scattered like small islands in the muscle of the neck and pyroninophilic cells were found infiltrating in the surrounding area. The recipient own kidney showed the same pathology as that in the transplanted kidney which is shown in Fig. 1 (Fig. 5). Apparently, no difference could be noticed between them. The liver showed a more chronic picture than the recipient liver in the 1st experimental group, degenerative changes, granuloma in the hepatic parenchyma, infiltration of pyroninophilic cells in the portal space, and abundant infiltration around the central vein were observed (Fig. 6, 7). 


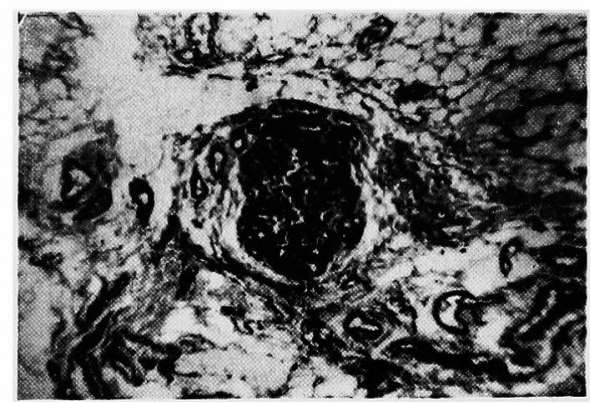

Fig. 4. Exp. No. DH 25. Homotransplanted kidney after 24 days. M-P staining. $\times 60$. Following necrosis and destruction of the transplanted kidney, it is buried and absorbed in the recipient muscle at neck.

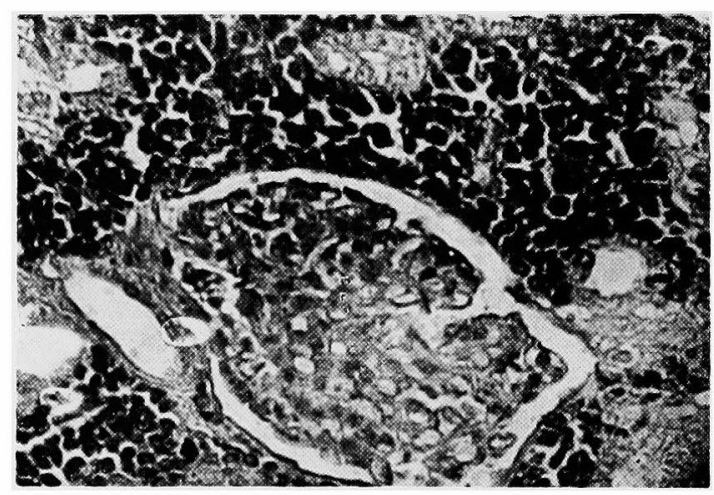

Fig. 5. Exp. No. DH 39. M-P staining. $\times 450$. Recipient kidney after 42 days. Periglomerular accumulation of the pyroninophilic cells.

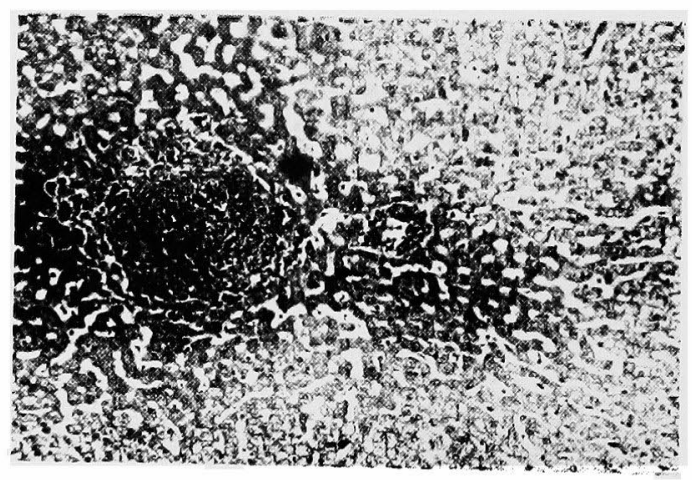

Fig. 6. Exp. No. DH 39. Recipient liver after 42 days. H-E staining. $\times 60$. Stasis, hyperaemia, central degeneration and necrosis. Granuloma is ovserved. 


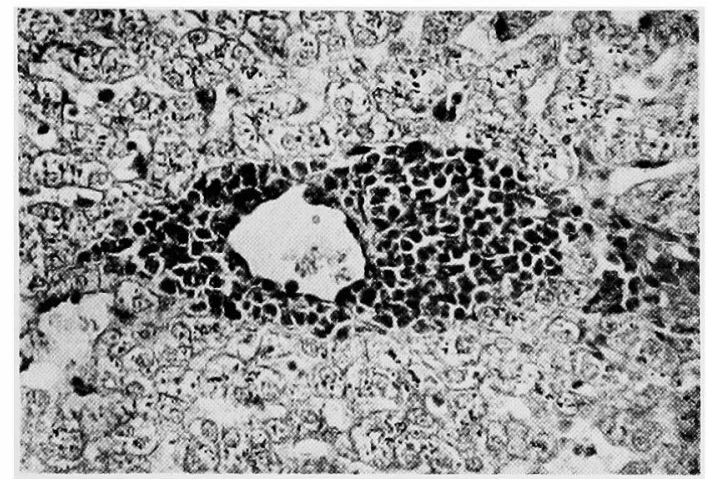

Fig. 7. Exp. No. DH 39. Recipient liver after 42 days. $\times 300$. Accumulation of pyroninophilic cells around central vein. Liver cells are degenerative.

\section{Discussion}

From the above experiment of two groups, interstitial nephritis which is accompanied with pyroninophilic cells will occur in the homotransplanted kidney from 2-8 days, and the kidney will become necrotic due to disturbance to the blood vessels, and eventually, it will be absorbed in the recipient, which is in accordance with other observations and leaves no doubt about it. However, it should be noticed that a similar changes will occur in the recipient kidney and liver as well as in the transplanted kidney. Reviewing the papers by Dempster and his associates ${ }^{51,10), 12)}$ and Simonsen et $a l^{7,111}$ who made an extensive experiment on homotransplantation of the kidney, respectively, the former writer did not mention anything on the changes which may occur in the recipient kidney and liver, while the latter described that " The liver and the heart showed no definite pathological changes- -". In our experiment with the first group, the transplanted kidney was circulated with the recipient blood for 2-8 days, and the recipient liver showed stagnation of blood, central degeneration, and infiltration of pyroninophilic cells around the blood vessels in the portal space. In the other experiment with group 2, in which the recipient kidney was left intact, a chronic pathological picture in the kidney could be studied several days later. It is a new observation that the recipient own kidney showed the same pathology as much as that in the transplanted kidney. The apathic and anorexic condition after tranplantation of the kidney as seen in dogs which belonged to group 1 and group 2, together with latent jaundice was not due to uremia, but may be regarded as hepatorenal disturbance of the recipient dog. The authors consider that the cause of hepatorenal disturbance in the recipient is as follows; Though the homotransplanted kidney will become necrotic due to the living responses 
of the recipient, the transplanted kidney acts as an independent individual as a "living activity unit". Consequently, the transplanted kidney and recipient will show the identical pathological picture. In other words, the transplanted kidney and recipient can act as an antigen to each other. The blood which circulates through both bodies will then continuously carry a large amount of antigen to each other, resulting abundant antibody production to these antigens, which will eventually cause progressive pathology in both dogs in a short time. The destruction of homotransplanted kidney may occur by the following mechanism. In our second experiment, despite destruction of the transplanted kidney, relatively chronic pathological finding with heavier cellular infiltration than that in the first experiment was observed in the recipient liver and kidney. This observation suggests that a pathology will occur in the recipient liver and kidney regardless the transplanted kidney is dead or alive. In the preceding paragraph, a word "living activity" was used, which menat that antigen was not an indispensable condition for the occurrence of homologous immunity phenomenon. It is, however, presumed that when transplanted kidney is living, a large amount of antigen is rapidly carried and immunity phenomenon is rapidly built up. Hitherto, it has been difficult to verify the immunity phenomenon between the same species. Pure immunological method, i.e., injection of kidney suspension into the homologous animals, could not produce the same pathological picture as that in the transplanted kidney, which may be due to interrupted administration of a small amount of antigen to the immunized recipient animal. Freund ${ }^{13)}$, Morgen ${ }^{14}$, and Kabat ${ }^{15)}$, respectively, performed homologous immunization by adding adjuvant to antigen, and were successful to produce organic pathological changes in the organs of the immunized recipient animal. By carrying out such pure immunological experiment, an effort to confirm the existence of homologous immunity phenomonon in homotransplantation of the kidney should be continued.

With the above-mentioned discovery of new informations, the syndrome observed in animals with transplanted kidneys which has been left as a question among those engaged in the work of homotransplantation of the kidney can be explained with ease. This toxic syndrome is consisted of 4 main manifestations, i.e. lethargy, anorexia, pyrexia and rapidly loss of weight. Following transplantation of the kidney, it occures in the recipient dramatic way, which weakens the recipient. Once the kidney ceases its function, it clears up spontaneously despite no care of the transplanted kidney. When the recipient is afflicted with this syndrome, it will recover dramatically if the transplanted kidney which is excreting urine is remov$\mathrm{ed}^{5,10)}$. However, if the recipient own kidney is left intact, the symptoms will recur remarkably, which is believed to be a curious syndrome which 
has no relationship with uremia. Dempster ${ }^{51,10)}$ reported that this syndrome occurred either in auto- or homo-transplantation of the kidney. Cultivating the transplanted kidney, B. coli, Ps. pyocyanea, and Staph. aureus were found, and bacterial infection was assumed its causative factor by him. He stated that terramycin had therapeutic effects for the prevention of occurrence. Later on, he reported that an unexplained changes which occurred in the transplanted kidney was the fundamental cause for such syndrome and attributed bacterial infection as a secondary phenomenon. In our experiment, this syndrome did not occur in the autotransplanted recipient kidney, but only in the homologous transplanted recipient kidney. Deklerk ${ }^{9}$ who found that the recipient was eosinopenic and hyponatremic claimed that the transplanted kidney became a stress to the recipient, resulting with occurrence of systemic toxic disturbance to the recipient. The author also observed that following transplantation of the kidney, the recipient became apathic, anorexic which lead to severe emaciation, whereas in group 2, when the transplanted kidney ceased to urinate, this syndrome ameliorated and lead the animal to a life as a normal one. As described earlier, once hepatorenal disturbance occurrs in the recipient, it may be natural that this syndrome would be observed. The reason that the syndrome improves following cease of renal function in the transplanted kidney is believed to be due to decrease of renal circulating blood volume secondary to destruction of the transplanted kidney, and the progress of hepatorenal disturbance in the recipient is slowed down by stop of living activity in the transplanted kidney, leading to spontaneous cure. As our explanation of this syndrome is a different opinion from those by Dempster et al.5),10),12), and Deklerk" followup study and comments from others are expected.

\section{SumMary}

We confirmed that after homologous transplantation of the kidney, similar pathological finding was observed in the recipient own liver and kidney as well as in the transplanted kidney. It is believed that systemic toxic syndrome in the recipient which has not been able to explain so far is due to such occurrence of pathology. No one has yet paid attention to this new information or has given due significance to it. Followup studies and comments are expected on this point.

\section{References}

1) Lawler, J. A. M. A., 1950, 144, 2, 844.

2) Murray, Am. J. Surg., 1954, 87, 4, 508.

3) Hume, J. Clin. Invest., 1955, 34, 327.

4) Merril, J. A. M. A., 1956, 160, 277. 
5) Dempster, Brit. J. Surg., 1953, 40, 327.

6) Oeconomos, Mem. Acad. Chir., 1953, 79, 642.

7) Simonsen, Acta Path. Microbiol. Scand., 1953, 32, 1.

8) Medawar, Brit. J. Exp. Path., 1946, 27, 9.

9) Deklerk, Ann. Surg., 1954, 140, 5, 711.

10) Dempster, Acta Med. Scand., 1952-53, 144, 360.

11) Simonsen, Acta Path. Microbiol. Scand., 1953, 32, 36.

12) Dempster, Ann. Roy, Coll, Surg. Eng., 1950, 7, 257.

13) Freund, Annual Review, Microbiol., 1947, 1, 291.

14) Morgan, J. Exp. Med., 1947, 85, 131.

15) Kabat, ibid., 1947, 85, 117. 